\title{
Optimal Solution of the Generalized Dubins Interval Problem
}

\author{
Petr Váňa, Jan Faigl
}

\begin{abstract}
The problem addressed in this paper is motivated by surveillance mission planning with curvature-constrained trajectories for Dubins vehicles that can be formulated as the Dubins Traveling Salesman Problem with Neighborhoods (DTSPN). We aim to provide a tight lower bound of the DTSPN, especially for the cases where the sequence of visits to the given regions is available. A problem to find the shortest Dubins path connecting two regions with prescribed intervals for possible departure and arrival heading angles of the vehicle is introduced. This new problem is called the Generalized Dubins Interval Problem (GDIP) and its optimal solution is addressed. Based on the solution of the GDIP, a tight lower bound of the above mentioned DTSPN is provided which is used to steer samplingbased algorithm to determine a feasible solution that is close to the optimum.
\end{abstract}

\section{INTRODUCTION}

Surveillance missions are frequent tasks for Unmanned Aerial Vehicles (UAVs) in which the vehicles are requested to visit a given set of target locations and collect the required data. The Dubins Traveling Salesman Problem (DTSP) [33] is one of the suitable problem formulations for planning such missions that respect the curvature-constrained trajectory of vehicles modeled as Dubins vehicle [5]. Two optimization parts can be identified in the DTSP: 1) the combinatorial optimization of the underlying TSP to determine the best sequence to visit the targets, and 2) the continuous optimization to determine the shortest path of the Dubins vehicle that is encoded by the vehicle heading angles at the target locations.

The DTSP is a challenging problem because the headings can be arbitrarily selected from the interval $[0,2 \pi)$ and it is known to be NP-hard [17] because of the underlying TSP. Therefore, the DTSP has been addressed by computationally efficient heuristic approaches that decouple the problem into the sequencing and the continuous optimization parts [33, 19, 21, 22, 36, 20, 14], or by simultaneous solution of both parts by unsupervised learning principles [6, 7]. In contrast, sampling-based [29, 28, 17, 31, 4] and evolutionary [35, 11] approaches are assumed to be resolution complete; but they are computationally demanding and unable to estimate the absolute quality of found solutions. The heuristic solution from [33] is proved to be bounded by the solution of the Euclidean TSP; however, the gap to the optimal solution is not tight, and it provides only a rough estimation of the solution quality. An approximation algorithm for the DTSP has been

The presented work has been supported by the Czech Science Foundation (GAČR) under research project No. 16-24206S.

Authors are with the Dept. of Computer Science, Czech Technical University in Prague, Technická 2, 16627 Prague, Czech Republic $\{$ vanapet 1 faiglj\}@fel.cvut.cz

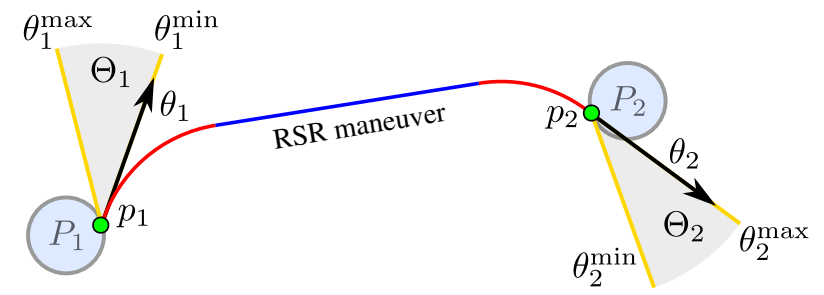

Fig. 1. GDIP instance $\left(P_{1}, \Theta_{1}, P_{2}, \Theta_{2}\right)$ and its solution that connects the regions $P_{1}$ and $P_{2}$ using the departure angle $\theta_{1} \in \Theta_{1}$ and the arrival angle $\theta_{2} \in \Theta_{2}$ at the particular locations $p_{1} \in P_{1}$ and $p_{2} \in P_{2}$.

proposed in [15] and further elaborated in [16] which improves the worst found solution especially for high-density instances.

A major step towards the optimal solution of the DTSP and a tight estimation of the solution quality has been made by introducing the Dubins Interval Problem (DIP) [27]. The DIP stands to find the shortest Dubins path between two endpoints with the heading angles restricted to be within the specified intervals. Based on the DIP, a tight lower bound of the DTSP has been proposed in [26, 27]. A sub-problem called the Dubins Touring Problem (DTP) with a given sequence of visits to the targets has been studied in [26]. Moreover, the solution of the DIP [27] has been employed to quickly find high-quality solutions of the DTP by steering sampling of the possible headings [8]. Such a solution can be utilized in other Dubins routing problems like the Dubins Orienteering Problem [32], where solutions are desirable to efficiently evaluate if the currently selected most rewarding targets fit into the limited travel budget.

The achievements enabled by the optimal solution of the DIP [27] motivate us to consider similar advancements for more practical surveillance missions where vehicles are allowed to save travel cost by covering target locations within a specified distance. Such missions can be formulated as the DTSP with Neighborhoods (DTSPN) [30] that has been addressed by similar methods [13, 10, 12, 23, 37, 34, 7] as for the DTSP.

Since the DIP cannot be directly utilized for the DTSPN, because of lack of the particular endpoints, we introduce the Generalized Dubins Interval Problem (GDIP) to find the shortest Dubins path between two regions with constrained heading angles at the endpoints, see an example in Fig. 1 The GDIP is theoretically analysed and optimal solution is proposed. Thus, the tight lower bound for the whole class of Dubins routing problems to visit a given set of regions is provided. The main contributions of the paper are considered as follows: 
1) The Generalized Dubins Interval Problem (GDIP).

2) The GDIP reduction to the One-Sided GDIP (OS-GDIP).

3) The optimal solution of the GDIP based on the transformation to the OS-GDIP.

4) Tight lower bound of the DTSPN with a given sequence of visits to the regions.

The rest of the paper is organized as follows. A brief description of the DIP and its properties are presented in the next section together with an overview of the related work. The proposed GDIP is introduced in Sec. III The transformation to the One-Sided GDIP (OS-GDIP) with the proof of its correctness is in Sec. IV] The optimal solution of the GDIP is proposed in Sec. V Benefits of the proposed GDIP is demonstrated in solving the DTSPN that are presented in Sec. VI, The final remarks are summarized in Sec. VII.

\section{The DIP AND RElated Work}

The Dubins vehicle is a curvature-constrained model defined by the constant forward speed $v$ and the minimum turning radius $\rho$. The state of the vehicle $q \in S E(2)$ is given by the position $p=(x, y)$ and the heading angle $\theta$. The model is

$$
\dot{q}=\left[\begin{array}{c}
\dot{x} \\
\dot{y} \\
\dot{\theta}
\end{array}\right]=v\left[\begin{array}{c}
\cos \theta \\
\sin \theta \\
\frac{u}{\rho}
\end{array}\right], \quad|u| \leq 1,
$$

where $u$ is the control input.

The shortest path between two configurations (further called Dubins maneuver [5]) is a combination of up to three straight segments (S) or arc segments (C) with the radius $\rho$, which results in two basic types of the maneuvers: CSC and CCC. The maneuver length is a piecewise continuous function with discontinuities at the boundary between these two basic types, and the transition may occur only if the endpoints are closer than four times $\rho$ (denoted as $\mathrm{D}_{4}$ ) [2, 3]; otherwise the CCC cannot be constructed.

The Dubins Interval Problem (DIP) stands to find the shortest Dubins maneuver from the state $q_{1}=\left(p_{1}, \theta_{1}\right)$ to $q_{2}=\left(p_{2}, \theta_{2}\right)$ while the heading angles $\theta_{1}, \theta_{2}$ are from the specified closed interval $\Theta_{1}, \Theta_{2}$ [27], i.e.,

Problem 2.1 (DIP):

$$
\begin{array}{ll} 
& \min _{\theta_{1}, \theta_{2}} \mathcal{L}\left(\left(p_{1}, \theta_{1}\right),\left(p_{2}, \theta_{2}\right)\right), \\
\text { s.t. } \quad & \theta_{1} \in \Theta_{1}, \theta_{2} \in \Theta_{2},
\end{array}
$$

where $\mathcal{L}(\cdot, \cdot)$ is the length of the Dubins maneuver between two configurations of the vehicle.

The DIP was solved in [24] using calculus and monotonicity properties of the Dubins maneuver and the properties of the DIP have been confirmed in [25] where the Pontryagin's minimum principle was utilized to prove the necessary conditions for the optimal solution. These conditions are summarized in Table \ and the maneuvers are divided into nine cases. In this paper, we follow the original notation [25] in which $\theta_{i}^{\text {min }}$ and $\theta_{i}^{\max }$ is the rightmost and leftmost heading angle, respectively. The subscript $\mathrm{L}_{\psi}$ denotes the angle of turn is greater than $\pi$.

\footnotetext{
${ }^{1} \mathrm{~L}_{\psi} \mathrm{R}_{\psi}$ and $\mathrm{R}_{\psi} \mathrm{L}_{\psi}$ types are claimed in 27] to be candidates to the optimal solution of the DIP, but they are not local minima.
}

\begin{tabular}{|c|c|c|c|}
\hline Case & Maneuvers & & Conditions on $\theta_{1}$ and $\theta_{2}$ \\
\hline 1) & $\mathrm{S}$ or $\mathrm{L}_{\psi}$ or $\mathrm{R}_{\psi} 1$ & & \\
\hline 2) & $\mathrm{LS}$ or $\mathrm{LR}_{\psi}$ & for & $\theta_{1}=\theta_{1}^{\max }$ and $\theta_{2} \in \Theta_{2}$ \\
\hline 3) & $\mathrm{RS}$ or $\mathrm{RL}_{\psi}$ & for & $\theta_{1}=\theta_{1}^{\min }$ and $\theta_{2} \in \Theta_{2}$ \\
\hline 4) & $\mathrm{SL}$ or $\mathrm{R}_{\psi} \mathrm{L}$ & for & $\theta_{1} \in \Theta_{1}$ and $\theta_{2}=\theta_{2}^{\min }$ \\
\hline 5) & $\mathrm{SR}$ or $\mathrm{L}_{\psi} \mathbf{R}$ & for & $\theta_{1} \in \Theta_{1}$ and $\theta_{2}=\theta_{2}^{\max }$ \\
\hline 6) & LSR & for & $\theta_{1}=\theta_{1}^{\max }$ and $\theta_{2}=\theta_{2}^{\max }$ \\
\hline 7) & $\mathrm{LSL}$ or $\mathrm{LR}_{\psi} \mathrm{L}$ & for & $\theta_{1}=\theta_{1}^{\max }$ and $\theta_{2}=\theta_{2}^{\min }$ \\
\hline 8) & RSL & for & $\theta_{1}=\theta_{1}^{\min }$ and $\theta_{2}=\theta_{2}^{\min }$ \\
\hline 9) & $\mathrm{RSR}$ or $\mathrm{RL}_{\psi} \mathrm{R}$ & for & $\theta_{1}=\theta_{1}^{\min }$ and $\theta_{2}=\theta_{2}^{\max }$ \\
\hline
\end{tabular}

TABLE I

LIST OF ALL POSSIBLY OPTIMAL SOLUTIONS OF THE DIP.

Based on the necessary conditions for the optimal solution of the DIP, the shortest path can be constructed separately for each particular case, if it exists. Then, the final solution is selected as the shortest one. Importantly, there exists a closed form expression for each particular case, because the first case contains only a single segment and at least one heading angle is fixed for the remaining types.

The DIP is a crucial building block for developing algorithms for more complex problems. In [27], it has been used for a tight lower bound estimation of the DTSP. The main idea is based on dividing possible headings at the targets into a set of heading intervals and transforming the problem into the Generalized Asymmetric TSP where particular distances are found as the solution of the corresponding instances of the DIP. The same idea is used for solving the DTSP with a given sequence of visits [26, 27], i.e., a solution of the DTP.

Approximation of the DTP is proposed in [18] and for the $\mathrm{D}_{4}$ constraint, the DTP is reducible to the limited number of convex optimization sub-problems [9]. The lower bound found by the DIP enables to estimate the solutions quality and steers the sampling process in an informative way to find high-quality solutions of the DTP much faster [8], e.g., finding solutions for 50 targets with the path length less than $1 \%$ from the optima in less than 10 seconds. The motivation of the proposed GDIP is to achieve similar results also for Dubins path visiting a sequence of target regions.

\section{Problem Statement (GDIP)}

The Generalized Dubins Interval Problem (GDIP) can be defined as follows. Let $P_{1}, P_{2}$ be compact regions in $\mathbb{R}^{2}$ and $\Theta_{1}, \Theta_{2}$ be closed intervals in $\mathbb{S}^{1}$. The GDIP stands to find the shortest Dubins maneuver from $P_{1}$ to $P_{2}$ such that the heading angles are within the specified closed intervals $\Theta_{1}, \Theta_{2}$.

Problem 3.1 (GDIP):

$$
\begin{gathered}
\min _{p_{1}, \theta_{1}, p_{2}, \theta_{2}} \mathcal{L}\left(\left(p_{1}, \theta_{1}\right),\left(p_{2}, \theta_{2}\right)\right), \\
\text { s.t. } \quad p_{1} \in P_{1}, \theta_{1} \in \Theta_{1}, p_{2} \in P_{2}, \theta_{2} \in \Theta_{2},
\end{gathered}
$$

where $\mathcal{L}(\cdot, \cdot)$ is the length of the corresponding Dubins maneuver connecting two configurations of the vehicle.

In general, the regions $P_{1}, P_{2}$ can be of any shape but in the rest of this paper the regions are restricted to the disks with the radii $r_{1}$ and $r_{2}$ centered at $c_{1}$ and $c_{2}$, respectively. Thus, for the endpoints $p_{1} \in P_{1}$ and $p_{2} \in P_{2}$, it holds

$$
\left\|p_{1}-c_{1}\right\| \leq r_{1}, \quad\left\|p_{2}-c_{2}\right\| \leq r_{2},
$$


where $\|\cdot\|$ is the Euclidean norm. An example of the GDIP instance and its solution is depicted in Fig. 11.

Note that in contrary to the DIP, the endpoints are not fixed in the GDIP, and they can be selected arbitrarily from the given regions, which significantly increases complexity of the problem. On the other hand, a solution of the GDIP enables to find a tight lower bound for a more general problem of the optimal Dubins path passing a sequence of regions, e.g., the DTSPN

A special version of the GDIP (further used to provide an optimal solution of the GDIP) is a degenerative case when the departure region $P_{1}$ is reduced to a single point $p_{1}^{\prime}$. Such a problem is called One-Sided GDIP (OS-GDIP).

Problem 3.2 (OS-GDIP):

$$
\begin{array}{cc} 
& \min _{\theta_{1}^{\prime}, p_{2}^{\prime}, \theta_{2}^{\prime}} \mathcal{L}\left(\left(p_{1}^{\prime}, \theta_{1}^{\prime}\right),\left(p_{2}^{\prime}, \theta_{2}^{\prime}\right)\right), \\
\text { s.t. } & \theta_{1}^{\prime} \in \Theta_{1}^{\prime}, p_{2}^{\prime} \in P_{2}^{\prime}, \theta_{2}^{\prime} \in \Theta_{2}^{\prime},
\end{array}
$$

where all the variables are distinguished (by a prime) from Problem 3.1 to clarify the OS-GDIP is transformed from the GDIP. This notation is used in the following analysis.

\section{ANALYSIS OF THE GDIP}

The GDIP is a continuous optimization problem minimizing the length of the Dubins maneuver with respect to the given regions and intervals of the heading angles. The endpoints of the maneuver are selected from the compact regions which can be considered as an extension of the existing DIP. Thus, the optimization problem has six degrees of freedom as $\mathcal{L}$ : $S E(2) \times S E(2) \rightarrow \mathbb{R}$. Two following ideas are utilized to reduce the complexity of the GDIP and provide its optimal solution.

First, the optimal solution of the GDIP is a path that is also the optimal solution of the corresponding DIP where the endpoints are fixed. This property is crucial because all the conditions for the DIP optimal solution summarized in Table I are directly applicable for the GDIP.

Secondly, the GDIP is reducible to the OS-GDIP, where one of the regions has zero radius, and thus the region is reduced to a single point. Such a transformation is possible because the GDIP is independent of the translation of its solution. The transformation is provided in the following paragraphs.

\section{A. Transformation of the GDIP to OS-GDIP and Vice-Versa}

The main assumption for transforming an GDIP instance $\mathcal{G}=\left(P_{1}, \Theta_{1}, P_{2}, \Theta_{2}\right)$ to a transformed OS-GDIP instance $\mathcal{G}^{\prime}=\left(p_{1}^{\prime}, \Theta_{1}^{\prime}, P_{2}^{\prime}, \Theta_{2}^{\prime}\right)$ is that any translation of the coordination system does not change the solution; the heading angles, the given intervals, and the maneuver length are all preserved. A solution of the original GDIP instance $\mathcal{G}$ is further denoted as $\tau=\left(p_{1}, \theta_{1}, p_{2}, \theta_{2}\right)$ and a solution of the transformed $\mathcal{G}^{\prime}$ as $\tau^{\prime}=\left(p_{1}^{\prime}, \theta_{1}^{\prime}, p_{2}^{\prime}, \theta_{2}^{\prime}\right)$. Therefore $\Theta_{1}=\Theta_{1}^{\prime}, \Theta_{2}=\Theta_{2}^{\prime}$ and also $\theta_{1}=\theta_{1}^{\prime}, \theta_{2}=\theta_{2}^{\prime}$, and thus we can focus only on the transformation of the endpoints and the regions.

To transform $\mathcal{G}$ into $\mathcal{G}^{\prime}$, a solution of $\mathcal{G}$ is translated such that it starts at the origin, which reduces the dimensionality

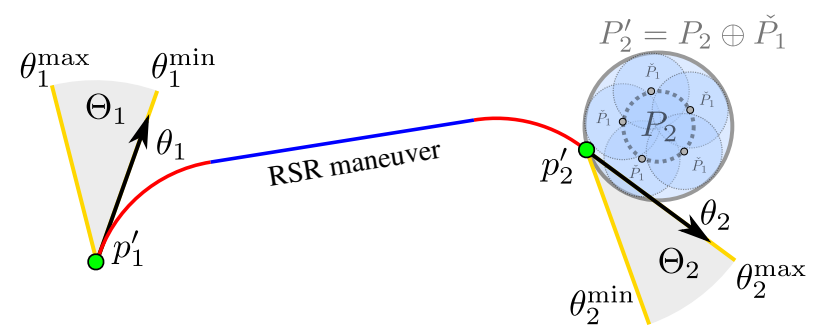

Fig. 2. OS-GDIP instance transformed from the GDIP instance in Fig. 1

of the problem because the region $P_{1}$ is reduced to a single point $p_{1}^{\prime}=(0,0)$. The transformed region $P_{1}^{\prime}$ contains only the origin and the region $P_{2}^{\prime}$ can be determined using the dilation operation $\oplus$ from the mathematical morphology, which is also known as the Minkowski sum,

$$
\begin{aligned}
& P_{1}^{\prime}=\left\{p_{1}^{\prime}\right\}=\{(0,0)\}, \\
& P_{2}^{\prime}=P_{2} \oplus \check{P}_{1}=\cup\left\{p_{b}-p_{a}, p_{a} \in P_{1}, p_{b} \in P_{2}\right\},
\end{aligned}
$$

where $\check{P}_{1}$ is the reflection of $P_{1}$, i.e., $\check{P}_{1}=\cup\left\{-p, p \in P_{1}\right\}$. An example of the OS-GDIP is shown in Fig. 2 .

Note the transformations (3) and (4) hold for regions of any shape as none of special properties are utilized. However, for the disk regions, the transformed $P_{2}^{\prime}$ is also a disk with the radius $r_{2}^{\prime}$ centered at $c_{2}^{\prime}$

$$
\begin{aligned}
r_{2}^{\prime} & =r_{1}+r_{2}, \\
c_{2}^{\prime} & =c_{2}-c_{1} .
\end{aligned}
$$

The forward transformation $\Phi_{12}$ of a feasible solution $\tau$ of the GDIP instance $\mathcal{G}$ to a feasible solution $\tau^{\prime}$ of the OS-GDIP instance $\mathcal{G}^{\prime}$ is a translation $\Phi_{12}(p): p \rightarrow p^{\prime}$ that moves the solution endpoint $p_{1}$ to $p_{1}^{\prime}=(0,0)$. The forward transformation is unique and it translates a point $p \in \mathbb{R}^{2}$ to the point $p^{\prime} \in \mathbb{R}^{2}$ by $-p_{1}$

$$
\Phi_{12}(p): \quad p \mapsto p-p_{1} .
$$

Lemma 4.1: Any feasible solution $\tau$ of the particular GDIP instance $\mathcal{G}$ can be transformed by $\Phi_{12}$ (7) into a feasible solution $\tau^{\prime}$ of the transformed OS-GDIP instance $\mathcal{G}^{\prime}$ created from $\mathcal{G}$.

Proof: To prove the lemma, the endpoints of the transformed solution (Dubins maneuver) have to be in the given regions, i.e., $p_{1}^{\prime} \in P_{1}^{\prime}$ and $p_{2}^{\prime} \in P_{2}^{\prime}$. The first is always correct by the definition (3). The second endpoint is transformed to $p_{2}^{\prime}=p_{2}-p_{1}$ and using (6) it results into $\left(p_{2}^{\prime}-c_{2}^{\prime}\right)=$ $\left(p_{2}-c_{2}\right)-\left(p_{1}-c_{1}\right)$. Using inequalities 2 for the original GDIP instance $\mathcal{G}$, the terminal position $p_{2}^{\prime}$ is constrained by $\left\|p_{2}^{\prime}-c_{2}^{\prime}\right\| \leq r_{2}+r_{1}$, and thus $p_{2}^{\prime} \in P_{2}^{\prime}$.

The backward transformation $\Phi_{21}$ is not unique and for a single feasible solution of the OS-GDIP instance $\mathcal{G}^{\prime}$, there may exist multiple solutions of the original GDIP instance $\mathcal{G}$. This ambiguity happens if $p_{2}^{\prime}$ is not at the boundary of $P_{2}^{\prime}$ because the original GDIP instance $\mathcal{G}$ has greater dimensionality than $\mathcal{G}^{\prime}$. Nevertheless, a universal formulation of the backward transformation $\Phi_{21}\left(p^{\prime}\right): p^{\prime} \rightarrow p$ exists as follows

$$
\Phi_{21}\left(p^{\prime}\right): \quad p^{\prime} \mapsto p^{\prime}+\left(c_{1}-\lambda\left(p_{2}^{\prime}-c_{2}^{\prime}\right)\right),
$$


where $\lambda$ represents the ratio of the region radii in the original instance $\mathcal{G}$

$$
\lambda=\frac{r_{1}}{r_{1}+r_{2}} .
$$

Lemma 4.2: Any feasible solution $\tau^{\prime}$ of the transformed OS-GDIP instance $\mathcal{G}^{\prime}$ can be transformed by $\Phi_{21}(8)$ into a feasible solution $\tau$ of the original GDIP instance $\mathcal{G}$.

Proof: Analogously to the forward transformation, the back-transformed solution needs to fulfill the original constraints (2). The first endpoint can be transformed back by $\Phi_{21}$ as $p_{1}=p_{1}^{\prime}+c_{1}-\lambda\left(p_{2}^{\prime}-c_{2}^{\prime}\right)$, where $p_{1}^{\prime}$ can be omitted because $p_{1}^{\prime}=(0,0)$. Knowing $\left\|p_{2}^{\prime}-c_{2}^{\prime}\right\| \leq r_{1}+r_{2}$ from (5) and by substituting it into the transformation $\Phi_{21}$ we get $\left\|p_{1}-c_{1}\right\| \leq r_{1}$, and thus $p_{1} \in P_{1}$. Similarly, for the second endpoint, $p_{2}=p_{2}^{\prime}+c_{1}-\lambda\left(p_{2}^{\prime}-c_{2}^{\prime}\right)$, and by subtracting $c_{2}=c_{2}^{\prime}+c_{1}$ from both sides of the equation, it results in $p_{2}-c_{2}=\left(p_{2}^{\prime}-c_{2}^{\prime}\right)(1-\lambda)$. Using (5), as for the first endpoint, it proves $\left\|p_{2}-c_{2}\right\| \leq r_{2}$, and thus $p_{2} \in P_{2}$.

Now, we need to show that the optimal solution of the transformed $\mathcal{G}^{\prime}$ is the optimal solution of the original $\mathcal{G}$.

Lemma 4.3: Let $\tau_{*}^{\prime}$ be an optimal solution of the transformed OS-GDIP instance $\mathcal{G}^{\prime}$, then the solution $\tau$ transformed back by $\Phi_{21}$ (8) is an optimal solution of the original GDIP instance $\mathcal{G}$, i.e., $\tau$ is $\tau_{*}$.

Proof: Let $\tau_{*}$ be an optimal solution of $\mathcal{G}$, then there exists an optimal solution $\tau_{*}^{\prime}$ of $\mathcal{G}^{\prime}$ with the same length because of Lemma 4.1. If $\tau_{*}^{\prime}$ is transformed back by $\Phi_{21}$ (8) it has the same length of $\tau_{*}$ because of Lemma 4.2, and thus it is an optimal solution of $\mathcal{G}$.

The main result of the forward (7) and backward (8) transformations is that any GDIP instance can be transformed to its one-sided version OS-GDIP. The transformation significantly reduces the search space of the original GDIP while all properties of the solution are preserved. Together with the DIP results [27], the OS-GDIP allows finding the optimal solution of the GDIP, which is proposed in the next section.

\section{Optimal SOLUTION OF THE GDIP}

The main challenge of finding the optimal solution of a GDIP instance is related to the determination of both departure and terminal configurations, i.e., $\left(p_{1}, \theta_{1}\right)$ and $\left(p_{2}, \theta_{2}\right)$, such that the length of the corresponding Dubins maneuver is minimized. The proposed solution is based on the transformation of the GDIP instance to the instance of the OS-GDIP using the transformations (3) and (4), which reduce the complexity of the problem as the first position $p_{1}$ of the GDIP becomes a single point in the OS-GDIP.

The OS-GDIP instance is solved using the necessary conditions for the optimal solution of the DIP summarized in Table II which clearly holds even for the GDIP and its subproblem OS-GDIP. However, it is still necessary to address the selection of the second endpoint $p_{2}^{\prime} \in P_{2}^{\prime}$, which needs to be found optimally. Therefore, each possible maneuver type is addressed separately because their geometrical properties differ significantly. Thus, multiple candidate solutions are found from which the shortest one is selected similarly as it is done for the DIP [27].
TABLE II

ALL POSSIBLY OPTIMAL SOLUTIONS OF THE GDIP

\begin{tabular}{lllr}
\hline Case & $\begin{array}{c}\text { Maneuver } \\
\text { type }\end{array}$ & \multicolumn{1}{c}{$\begin{array}{c}\text { All possible } \\
\text { maneuvers }\end{array}$} & $\begin{array}{c}\text { Closed-form } \\
\text { solution }\end{array}$ \\
\hline 1) & $\mathrm{S}$ & $\mathrm{S}$ & YES \\
2) & $\mathrm{CS}$ & $\mathrm{L}, \mathrm{R}, \mathrm{LS}, \mathrm{RS}, \mathrm{SL}, \mathrm{SR}$ & YES \\
$3)$ & $\mathrm{C}_{\psi}$ & $\mathrm{L}_{\psi}, \mathrm{R}_{\psi}$ & YES \\
$4)$ & $\mathrm{CSC}$ & $\mathrm{LSL}, \mathrm{RSR}$ & YES \\
5) & $\mathrm{CS} \overline{\mathrm{C}}$ & $\mathrm{LR}, \mathrm{RL}, \mathrm{LSR}, \mathrm{RSL}$ & YES \\
$6)$ & $\mathrm{C} \overline{\mathrm{C}}_{\psi} \mathrm{C}$ & $\mathrm{LRL}, \mathrm{RLR}$ & YES \\
$7)$ & $\mathrm{C}_{\psi}$ & $\mathrm{LR}_{\psi}, \mathrm{RL}_{\psi}, \mathrm{L}_{\psi} \mathrm{R}, \mathrm{R}_{\psi} \mathrm{L}$ & $\mathrm{NO}$ \\
\hline
\end{tabular}

Finally, the optimal solution of the OS-GDIP instance is transformed to the solution of the original GDIP instance using the backward transformation (8). Since neither of the transformations changes the solution or its length, the proposed approach always finds the optimal solution. For the rest of this section, we provide a solution of the OS-GDIP instance.

\section{A. Solving the OS-GDIP for Specific Types of Maneuvers}

A solution of the GDIP follows the theoretical results of the DIP, and there exist 20 possibly optimal GDIP maneuvers that are all listed in Table III All maneuvers are categorized into seven basic types according to their geometrical properties. The original notation of the DIP is slightly modified and the arc segment is denoted by $\mathrm{C}_{\psi}$ if the turning angle is greater than $\pi$, but $\overline{\mathrm{C}}$ stands for an opposite turning direction. This modification enables to distinguish between CSC in which both arc segments have the same orientation and $\operatorname{CS} \bar{C}$ for the opposite orientation, because the method of finding the optimal solution differs. For each type of the maneuvers, the optimal solution is provided separately.

The following notation is used in the rest of this section. The symbol $\mathcal{L}$ with a subscript defining the maneuver type denotes the optimal length of such Dubins maneuver. Particular segments of the same type in a single maneuver are distinguished by the subscript defining the order of the segment in the maneuver, e.g., $\mathrm{C}_{1}$ is the first $\mathrm{C}$ segment of the CSC maneuver. An angle defined by two points $p_{i}, c_{j}$ is denoted $\angle p_{i} c_{j}$ and analogously for three points.

1) $\mathbf{S}$ maneuver: The simplest maneuver contains a single straight segment $\mathrm{S}$ and its direction is given by the angle $\theta_{\mathrm{S}}$ at the intersection $\Theta_{S}$ of the both given intervals

$$
\theta_{\mathrm{S}} \in \Theta_{\mathrm{S}}, \quad \Theta_{\mathrm{S}}=\Theta_{1} \cup \Theta_{2},
$$

where $\Theta_{\mathrm{S}}$ may contain up to two intervals. If the $\mathrm{S}$ maneuver is the optimal solution, one of the following cases occurs:

1.a) If $p_{1}^{\prime} \in P_{2}^{\prime}$ and $\Theta_{\mathrm{S}} \neq \emptyset$ then $\mathcal{L}_{\mathrm{S}}=0$.

1.b) If the above does not hold and $\angle p_{1}^{\prime} c_{2}^{\prime} \in \Theta_{\mathrm{S}}$ then $\theta_{\mathrm{S}}=$ $\angle p_{1}^{\prime} s_{2}^{\prime}$ and $\mathcal{L}_{\mathrm{S}}=\left\|p_{1}^{\prime}-c_{2}^{\prime}\right\|-r_{2}^{\prime}$.

1.c) If the above do not hold and $\exists p_{2}^{\prime}: p_{2}^{\prime} \in P_{2}^{\prime}$ such that $\angle p_{1}^{\prime} s_{2}^{\prime} \in \Theta_{\mathrm{S}}$; then $\theta_{\mathrm{S}}$ is at the boundaries of $\Theta_{\mathrm{S}}$.

All of the cases can be found using basic algebra operations and an example of the $1 . b$ case is shown in Fig. 3 . 


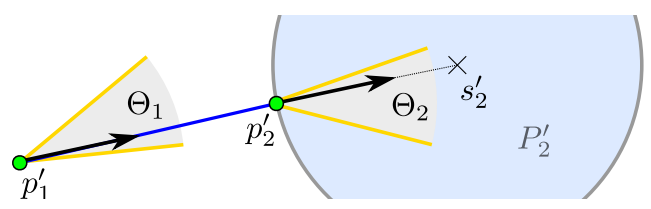

Fig. 3. An example of the straight maneuver solution of the OS-GDIP.

2) CS maneuvers: The next possible candidates for the optimal solution are the maneuvers of the CS type with a non-zero arc segment to differentiate it from the previous case. There are six possible combinations of maneuvers $\{\mathrm{R}, \mathrm{L}, \mathrm{LS}$, RS, SL, SR $\}$ of the CS type, but only the R and RS maneuvers are analyzed as the problem is symmetric and can be addressed as follows. For the combinations SL and SR, an orientation of the solution is reverted and the original instance of the GDIP is transformed such that $r_{2}^{\prime}=0$ and $r_{1}^{\prime}=r_{1}+r_{2}$. Further, both $\Theta_{1}$ and $\Theta_{2}$ are reverted, and thus the problem is transformed to a different instance of the OS-GDIP where the solution is the LS or RS maneuver.

For the R and RS maneuvers, the departure angle $\theta_{1}=\Theta_{1}^{\min }$ is a priory known. Thus, the center $o_{1}$ of the first arc segment is fixed and it remains to choose the terminal angle such that $\theta_{2} \in \Theta_{2}$ and the length $\mathcal{L}_{\mathrm{RS}}$ is minimized. Let the length of the circle segment be $\mathcal{L}_{\mathrm{R}}>0$ and $\mathcal{L}_{\mathrm{S}}$ be the length of the straight segment. Then, three following cases can occur:

2.a) The terminal angle is $\theta_{2}=\Theta_{2}^{\max }$ and $\mathcal{L}_{\mathrm{S}}=0$.

2.b) If $\left\|o_{1}-c_{2}^{\prime}\right\| \leq \rho$; the terminal point $p_{2}^{\prime}$ is at the intersection between the arc segment and $P_{2}^{\prime}$, and thus $\mathcal{L}_{\mathrm{S}}=0$.

2.c) If $\left\|o_{1}-c_{2}^{\prime}\right\|>\rho$ then $\mathcal{L}_{\mathrm{S}}>0$ and the extension of the straight segment passes through $c_{2}^{\prime}$.

A closed-form solution exists for all three cases. The first two cases 2.a and 2.b are special boundary cases, which are obviously local optima; however, the optimality of the last case $2 . c$ is proved in the following lemma.

Lemma 5.1: If the optimal solution of the OS-GDIP instance is the RS maneuver and $\mathcal{L}_{\mathrm{S}}>0$, a line extension of the straight segment passes through $s_{2}^{\prime}$ (the center of $P_{2}^{\prime}$ ).

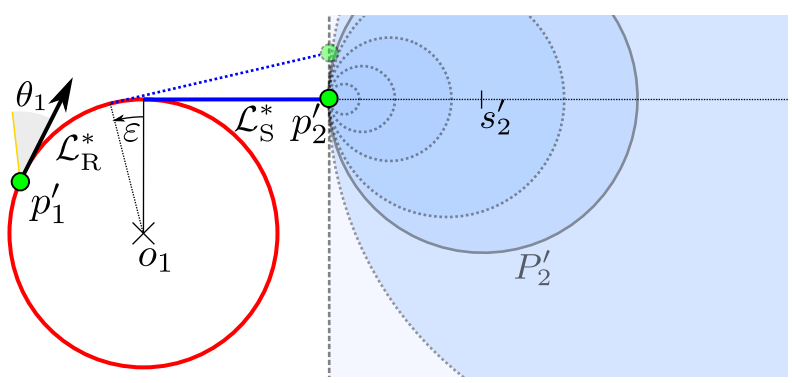

Fig. 4. RS maneuver of the CS type for various radii of $P_{2}^{\prime}$ depicted as the blue disks which become the blue half-plane in the limit case.

Proof: Let $\mathcal{L}_{\mathrm{R}}^{*}$ be the optimal length of the R segment, $\mathcal{L}_{\mathrm{S}}^{*}$ be the optimal length of the $\mathrm{S}$ segment, and $\varepsilon$ be the deviation from the direction towards $c_{2}^{\prime}$ (the center of $P_{2}^{\prime}$ ), see Fig. 4 . Supposing the optimal solution meets the lemma, the worst possible case is when the radius of the region $P_{2}^{\prime}$ goes to infinity. Thus, $P_{2}^{\prime}$ becomes a half-plane which minimizes the length of all other feasible maneuvers for which $\varepsilon \neq 0$. The total length of such a maneuver is

$$
\mathcal{L}_{\mathrm{RS}}=\frac{\mathcal{L}_{\mathrm{S}}^{*}+\rho \sin (\varepsilon)}{\cos (\varepsilon)}+\mathcal{L}_{\mathrm{R}}^{*}-\rho \varepsilon
$$

The derivative of the length function with respect to $\varepsilon$ can be expressed as

$$
\frac{\partial}{\partial \varepsilon} \mathcal{L}_{\mathrm{RS}}=\tan (\varepsilon) \sec (\varepsilon)\left(\mathcal{L}_{\mathrm{S}}^{*}+\rho \sin (\varepsilon)\right)
$$

The RS maneuver can occur only if $\varepsilon \in(-\pi, \pi)$ and $\mathcal{L}_{\mathrm{S}}^{*}+$ $\rho \sin (\varepsilon)>0$; otherwise $\mathcal{L}_{\mathrm{S}} \ngtr 0$. The only local optimum occurs for $\varepsilon=0$, and thus a line extension of the $\mathrm{S}$ segment passes thought $c_{2}^{\prime}$.

3) $\mathbf{C}_{\psi}$ maneuvers: If $p_{1}^{\prime}$ and $P_{2}^{\prime}$ are close enough, maneuvers of the $\mathrm{C}_{\psi}$ type may become the optimal solution. The boundary cases of the heading angles are already covered by the CS type of the maneuvers, and therefore, such cases are forbidden for the $\mathrm{C}_{\psi}$ type of maneuvers, which can be expressed as

$$
\theta_{1} \in \Theta_{1} \backslash\left\{\theta_{1}^{\min }, \theta_{1}^{\max }\right\}, \quad \theta_{2} \in \Theta_{2} \backslash\left\{\theta_{2}^{\min }, \theta_{2}^{\max }\right\} .
$$

Lemma 5.2: If the optimal solution of the OS-GDIP instance is of the $\mathrm{C}_{\psi}$ type, then $p_{2}^{\prime}=\max _{p \in P_{2}^{\prime}}\left\|p-p_{1}^{\prime}\right\|$.

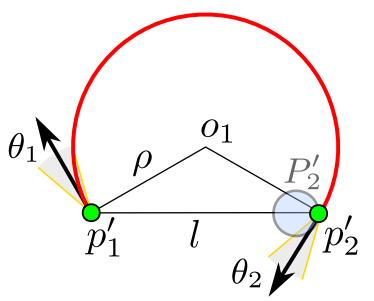

Fig. 5. $\mathbf{R}_{\psi}$ maneuver of the $\mathrm{C}_{\psi}$ type in the solution of the OS-GDIP.

Proof: Let $\mathcal{L}_{\mathrm{C}_{\psi}}$ be the length of the $\mathrm{C}_{\psi}$ maneuver which depends on the distance $l=\left\|p_{1}^{\prime}-p_{2}^{\prime}\right\|$, see Fig. 5 Then

$$
\mathcal{L}_{\mathrm{C}_{\psi}}=\rho\left(2 \pi-2 \arcsin \left(\frac{l}{2 \rho}\right)\right) .
$$

The length $\mathcal{L}_{\mathrm{C}_{\psi}}$ depends only on the distance $l$ and it is independent on the specific $p_{2}^{\prime}$ location and its derivative can be expressed as

$$
\frac{\partial}{\partial l} \mathcal{L}_{\mathrm{R}_{\psi}}=\frac{-2}{\sqrt{4-\frac{l^{2}}{\rho^{2}}}} .
$$

The derivative is always negative for the cases in which a maneuver of the $\mathrm{C}_{\psi}$ type can be constructed, i.e., for $l<2 \rho$. Thus, the terminal position $p_{2}^{\prime}$ of the optimal maneuver of the $\mathrm{C}_{\psi}$ type is such that the value of $l$ is maximized.

Notice the maneuver of the $\mathrm{C}_{\psi}$ type can be optimal only if the farthermost $p_{2}^{\prime}$ location meets $l<2 \rho$; otherwise there exists a shorter maneuver of a different type. Therefore, the necessary condition that a maneuver of the $\mathrm{C}_{\psi}$ type can be optimal is $\left\|p_{1}^{\prime}-s_{2}^{\prime}\right\|+r_{2}^{\prime}<2 \rho$. 
4) CSC maneuvers: Maneuvers of the CSC type contain two $C$ segments $C_{1}$ and $C_{2}$ with the same orientation connected by the central $\mathrm{S}$ segment. All three segments are considered to have a non-zero length; otherwise the maneuver reduces into one of the above cases. Both the heading angles are known to be at the limits of the intervals:

$$
\operatorname{CSC}\left\{\begin{array}{l}
\text { LSL : } \theta_{1}=\theta_{1}^{\max } \wedge \theta_{2}=\theta_{2}^{\min } \\
\operatorname{RSR}: \theta_{1}=\theta_{1}^{\min } \wedge \theta_{2}=\theta_{2}^{\max } .
\end{array}\right.
$$

The total length of the $\mathrm{C}$ maneuvers $\left(\mathcal{L}_{\mathrm{C}_{1}}+\mathcal{L}_{\mathrm{C}_{2}}\right)$ is given by $\theta_{1}, \theta_{2}$. However, the optimal position of $p_{2}^{\prime}$ needs to be found. Let $p_{2}^{\prime \prime}$ be a virtual endpoint of the maneuver of the CSC type, where the central S segment is omitted, see Fig. 6. Then, the following lemma can be formulated.

Lemma 5.3: If the optimal solution of the OS-GDIP instance is a maneuver of the CSC type and all three segments have non-zero length, then $p_{2}^{\prime}=\min _{p \in P_{2}^{\prime}}\left\|p-p_{2}^{\prime \prime}\right\|$.

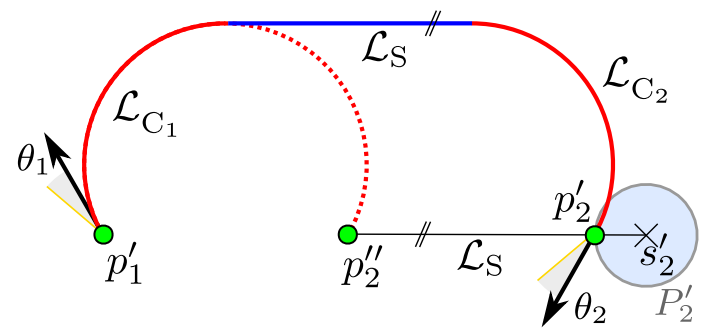

Fig. 6. RSR maneuver of the CSC type in the solution of the OS-GDIP.

Proof: Since the sum of $\mathcal{L}_{\mathrm{C}_{1}}$ and $\mathcal{L}_{\mathrm{C}_{2}}$ is a priory known, the total length is influenced only by the length $\mathcal{L}_{\mathrm{S}}$ of the straight segment. The orientation of the $\mathrm{S}$ segment is parallel to $p_{2}^{\prime \prime} p_{2}^{\prime}$ and $\mathcal{L}_{\mathrm{S}}=\left\|p_{2}^{\prime \prime}-p_{2}^{\prime}\right\|$. Therefore, $\mathcal{L}_{\mathrm{S}}$ needs to be minimized to get the optimal solution.

5) CS $\overline{\mathbf{C}}$ maneuvers: Maneuvers of the CS $\bar{C}$ type contain two arc segments with the opposite orientations and the center S segment which may have zero length. Similarly to the previous case, the optimal heading angles are a priory known:

$$
\operatorname{CS} \bar{C}\left\{\begin{array}{l}
\text { LSR : } \theta_{1}=\theta_{1}^{\max } \wedge \theta_{2}=\theta_{2}^{\max }, \\
\operatorname{RSL}: \theta_{1}=\theta_{1}^{\min } \wedge \theta_{2}=\theta_{2}^{\min } .
\end{array}\right.
$$

In contrast to the previous CSC type, this type of maneuvers is more complex because the lengths of the arc segments cannot be determined directly from $\theta_{1}, \theta_{2}$. Therefore, a transformation to a different OS-GDIP instance is considered.

Lemma 5.4: If the optimal solution of the OS-GDIP is a maneuver of the CSC type, the problem instance can be transformed into a OS-GDIP instance where the solution is of the CS type.

Proof: Since $\theta_{1}, \theta_{2}$ are fixed, the center $o_{1}$ of the first $\mathrm{C}$ segment is known and the second center $O_{2}$ lies inside the disk region $\mathrm{O}_{2}$ which corresponds to $P_{2}^{\prime}$ translated by $\rho$ perpendicularly to $\theta_{2}$, see Fig. 7. Let $t$ be the center of the $\mathrm{S}$ segment, then $t$ is also a midpoint between $o_{1}$ and $o_{2}$ because of the central symmetry of the maneuver. Based on $o_{1}$ and $\mathrm{O}_{2}$, a set of all possible $t$ positions is a circle region $T$ with

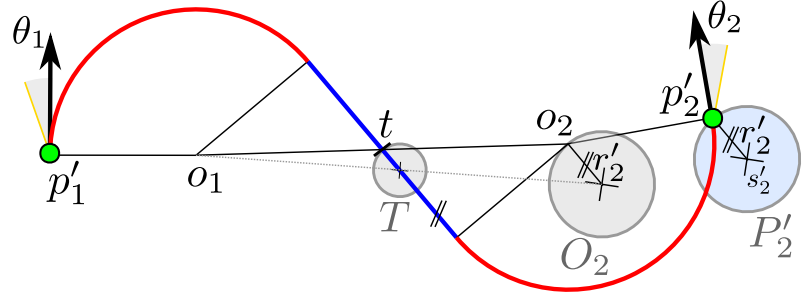

Fig. 7. RSL maneuver of the CSC type in the solution of the OS-GDIP.

the center at the midpoint between $o_{1}$ and $O_{2}$ center and with the radius $r_{2}^{\prime}$. Thus, the problem is transformed into finding a maneuver of the CS type from $\left(p_{1}, \theta_{1}\right)$ into the region $T$ without any constraint on the terminal angle. This defines a different OS-GDIP instance in which a maneuver of the CS type is to be found.

As a result of Lemma 5.4 the problem of finding a maneuver of the CSC type is transformed into a less complex problem for which a closed-form solution exists as it is shown above.

6) $\mathbf{C} \overline{\mathbf{C}}_{\psi} \mathbf{C}$ maneuvers: If $\left\|p_{1}^{\prime}-p_{2}^{\prime}\right\|<4 \rho$, the maneuvers of the $\mathrm{C}_{\psi} \mathrm{C}$ type can be the optimal solution because one of the maneuvers of the CST type cannot be constructed. The optimal heading angles are a priory known

$$
\mathrm{CC}_{\psi} \mathrm{C}\left\{\begin{array}{l}
\mathrm{LR}_{\psi} \mathrm{L}: \theta_{1}=\theta_{1}^{\max } \wedge \theta_{2}=\theta_{2}^{\min }, \\
\mathrm{RL}_{\psi} \mathrm{R}: \theta_{1}=\theta_{1}^{\min } \wedge \theta_{2}=\theta_{2}^{\max } .
\end{array}\right.
$$

Similarly to the CSC type of maneuvers, the center $o_{1}$ of the first $\mathrm{C}$ segment is known and the third center $o_{3}$ lies inside the disk region $\mathrm{O}_{3}$ derived from $P_{2}^{\prime}$, see Fig. 8 Then, the third center $o_{3}$ is determined based on the following lemma.

Lemma 5.5: If the optimal solution of the OS-GDIP is a maneuver of the $\mathrm{C}_{\psi} \mathrm{C}$ type, then $o_{3}=\max _{s \in O_{3}}\left\|s-o_{1}\right\|$.

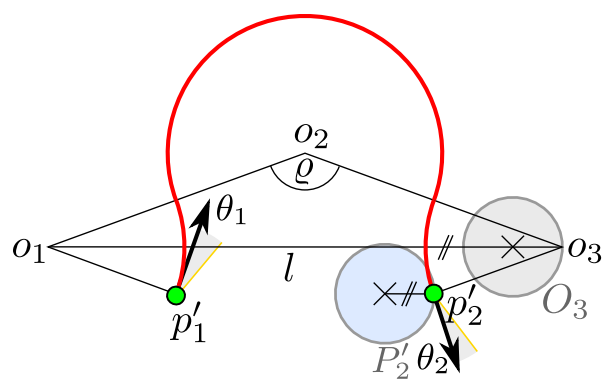

Fig. 8. LRL maneuver of the $\mathrm{C} \overline{\mathrm{C}}_{\psi} \mathrm{C}$ type in the solution for the OS-GDIP.

Proof: Since $\theta_{1}, \theta_{2}$ are fixed, the length of the maneuver can be determined based on the angle $\varrho=\angle \mathrm{O}_{1} \mathrm{O}_{2} \mathrm{O}_{3}$

$$
\mathcal{L}_{\mathrm{C}_{\psi} \mathrm{C}}= \pm\left(\theta_{1}-\theta_{2}\right)-2 \varrho+2 k \pi
$$

where $k \in \mathbb{Z}$ is for the angle normalization. Therefore $\varrho$ needs to be maximized to get the optimal solution which causes the distance $l=\left\|o_{1}-o_{3}\right\|$ is maximized.

Notice $o_{3}$ can be found by a closed-form expression because $O_{3}$ is a circular region. Having $p_{1}$ and $p_{3}$, the maneuver is well defined and easy to construct. 
7) $\mathbf{C} \overline{\mathbf{C}}_{\psi}$ maneuvers: The last type of maneuvers is $\bar{C}_{\psi}$ which can also occurs only if $\left\|p_{1}^{\prime}-p_{2}^{\prime}\right\|<4 \rho$. There may exist up to four maneuvers for which one of the heading angles is known:

$$
\mathbf{C C}_{\psi}\left\{\begin{array}{l}
\overline{\mathbf{L}}_{\psi}: \theta_{1}=\theta_{1}^{\max } \wedge \theta_{2} \in \Theta_{2}, \\
\mathrm{R}_{\psi}: \theta_{1}=\theta_{1}^{\min } \wedge \theta_{2} \in \Theta_{2}, \\
\overline{\mathbf{R}}_{\psi} \mathbf{L}: \theta_{1} \in \Theta_{1} \wedge \theta_{2}=\theta_{2}^{\min }, \\
\overline{\mathbf{L}}_{\psi} \mathbf{R}: \theta_{1} \in \Theta_{1} \wedge \theta_{2}=\theta_{2}^{\max } .
\end{array}\right.
$$

Only the maneuver $\mathrm{L} \overline{\mathrm{R}}_{\psi}$ of the $\mathrm{C} \overline{\mathrm{C}}_{\psi}$ type is further studied because other maneuvers share the same properties. Let $o_{1}$ and $O_{2}$ be the centers of the $\mathrm{C}$ segments, then the length of the maneuver depends on $p_{2}^{\prime}$ :

$$
\mathcal{L}_{\mathrm{L}_{\psi}}=\rho(2 \pi-\alpha+\beta+\varphi),
$$

where $\varphi=\angle p_{1}^{\prime} o_{1} p_{2}^{\prime}$ and $\alpha=\angle o_{1} o_{2} p_{2}^{\prime}, \beta=\angle p_{2}^{\prime} o_{1} o_{2}$ which depends on the distance $d=\left\|o_{1}-p_{2}^{\prime}\right\|$, see Fig. 9 . The maneuver length from (21) can be expressed in the polar

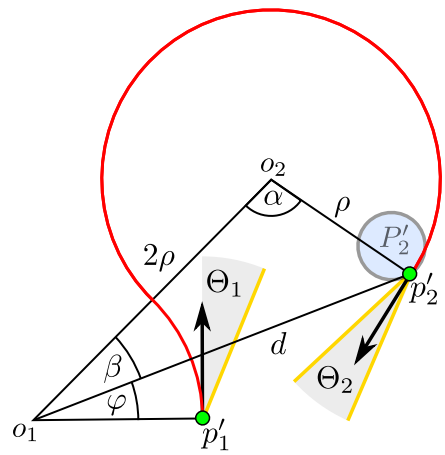

Fig. 9. $L \bar{R}_{\psi}$ maneuver of the $C \bar{C}_{\psi}$ type in the solution of the OS-GDIP.

coordination system of $(\psi, d)$ as

$$
\begin{aligned}
\mathcal{L}_{\mathrm{L} \overline{\mathrm{R}}_{\psi}}=\rho\left[2 \pi-\cos ^{-1}\right. & \left(\frac{5 \rho^{2}-d^{2}}{4 \rho^{2}}\right) \\
& \left.+\cos ^{-1}\left(\frac{3 \rho^{2}+d^{2}}{4 d \rho}\right)+\varphi\right] .
\end{aligned}
$$

The derivatives of the maneuver length are

$$
\frac{\partial}{\partial \varphi} \mathcal{L}_{\mathrm{LR}_{\psi}}=\rho, \quad \frac{\partial}{\partial d} \mathcal{L}_{\mathrm{LR}_{\psi}}=\frac{3 \rho^{2}-3 d^{2}}{d \sqrt{-d^{4}+10 d^{2} \rho^{2}-9 \rho^{4}}}
$$

From the domain of the second derivative according to $d$, the necessary condition for the $\mathrm{C} \overline{\mathrm{C}}_{\psi}$ maneuver to exist is

$$
\rho \leq d \leq 3 \rho,
$$

which corresponds to a direct geometrical representation. Thus, the signs of both derivatives are fixed

$$
\frac{\partial}{\partial \varphi} \mathcal{L}_{\mathrm{LR}_{\psi}}>0, \quad \frac{\partial}{\partial d} \mathcal{L}_{\mathrm{L} \overline{\mathrm{R}}_{\psi}}<0 .
$$

Based on these preliminaries, the optimality of the maneuver is shown in Lemma 5.6 .
Lemma 5.6: If the optimal solution of the OS-GDIP instance is the $\mathrm{C} \overline{\mathrm{C}}_{\psi}$ maneuver, the terminal position $p_{2}^{\prime}$ lies at the boundary of the departure region $P_{2}^{\prime}$.

Proof: The sings of both length derivatives are fixed, and thus the minimum of the length function is at the boundary of $P_{2}^{\prime}$ or a maneuver of the $\mathrm{C} \overline{\mathrm{C}}_{\psi}$ type is not optimal.

Notice the second derivative of the $\mathcal{L}_{\mathrm{LR}_{\psi}}$ over $d$ can be expressed as

$$
\frac{\partial^{2}}{\partial d} \mathcal{L}_{\mathrm{L} \overline{\mathrm{R}}_{\psi}}=\frac{3\left(9 \rho^{4}-2 \rho^{2} d^{2}+d^{4}\right)}{d^{2}\left(d^{2}-9 \rho^{2}\right) \sqrt{-9 \rho^{4}+10 \rho^{2} d^{2}-d^{4}}},
$$

for which $\frac{\partial^{2}}{\partial d} \mathcal{L}_{\mathrm{L} \overline{\mathrm{R}}_{\psi}}<0$ holds if $d$ is in the interval from 24. The second derivative over $\psi$ is always zero, i.e., $\frac{\partial^{2}}{\partial \psi} \mathcal{L}_{\mathrm{LR}}=0$.

Knowing one of the second derivatives is zero and the other derivative is always negative, the problem can be seen as an optimization problem which contains a single minimum. Furthermore, the position of $p_{2}^{\prime}$ in the optimal solution is known to be at the boundary of $P_{2}^{\prime}$, which reduces the problem to a convex optimization problem with a single variable. A close-form formula is not known but the problem can be easily solved by a numerical method, e.g., hill-climbing.

\section{B. Implementation of the optimal solution}

An algorithm evaluating the above-presented cases for finding the optimal solution of the GDIP has been implemented in $\mathrm{C}++{ }^{2}$ For the first six types of the maneuvers, a closed-form solution exists and it can be implemented using trigonometric functions. However, for the last type of the $\mathrm{C} \overline{\mathrm{C}}_{\psi}$ maneuver, no closed-form solution has been found, and therefore, it is solved as a convex optimization problem with a single variable. Based on the implementation, the required computational time of the optimal solution of the GDIP is about 22 times slower than a single determination of the Dubins maneuver for fixed configurations. The achieved average computation times from one million randomly generated GDIP instances are reported in Table III to provide an overview of the real computational requirements.

TABLE III

\begin{tabular}{lcc}
\multicolumn{3}{c}{ AVERAGE COMPUTATIONAL TIME PER ONE SOLUTION } \\
\hline Problem & Time $[\mu \mathrm{s}]$ & Ratio to DM \\
\hline Dubins Maneuver (DM) & 0.58 & 1.00 \\
DIP & 2.86 & 4.93 \\
GDIP & 12.63 & 21.78 \\
\hline
\end{tabular}

Note that all the results reported in this paper have been computed using a single core of the Intel Core i5-7600K CPU running at $3.8 \mathrm{GHz}$.

\section{GDIP IN SOLUTION OF THE DTSPN}

The main motivation of the proposed GDIP is to provide tight lower bound of the problem to find a shortest Dubins path through a sequence of the given target regions. Moreover, the lower bound can be utilized in finding high-quality solutions of the DTSPN similarly as the DIP is employed in the solution of the DTP and DTSP [26, 27, 8]. Therefore, we consider the

\footnotetext{
2 The source codes are publicly available at https://github.com/comrob/gdip
} 
GDIP in the solution of the DTSPN for which the sequence of visits to the disk-shaped target regions is determined as a solution of the Euclidean TSP (ETSP) using the centers of the disks. The DTSPN with a sequence of visits is called the Dubins Touring Regions Problem (DTRP) to distinguish the sequence is given or found independently on the continuous optimization part of the DTSPN.

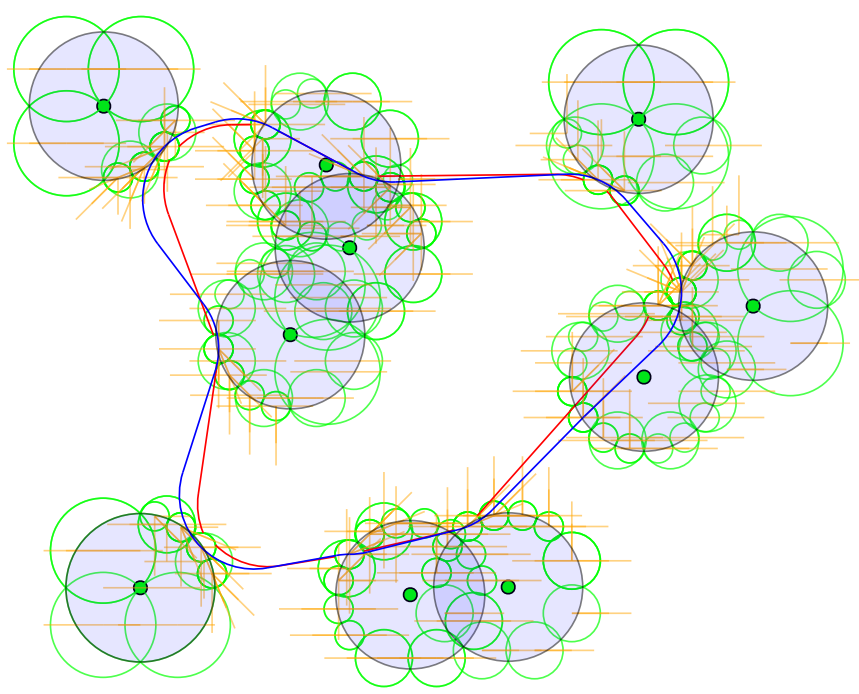

Fig. 10. An example of the DTRP instance with $n=10$ target regions with the radius $\delta=1$ (visualized as light blue disks) and $\rho=1$. A lower bound path is 17.68 long (red) and a feasible solution is 22.07 long (blue) both determined in $0.66 \mathrm{~s}$ for $k_{\max }=16$. The green circles are the samples and the orange lines correspond to intervals of the heading angles.

The solution of the DTRP follows the idea of the DTP [8] and samples the configuration space of the vehicle. Each sample of the target region consists of a circular sub-region and interval of the heading angle. The sampled circular subregions are located on the boundary of the region because the final Dubins path has to intersect the boundary of each target region, see example of the solution with the samples, lower bound, and feasible solutions in Fig. 10

The samples are iteratively refined to find a feasible solution tightly bounded by the lower bound solution provided by the introduced GDIP. This procedure ends when maximum resolution of $k_{\max }$ samples (per each region) is reached. Then, a feasible solution is found by forward search using the samples selected from the lower bound solution.

A full description of the algorithm is out of the scope of this paper due to the page limit. Therefore, a brief overview is provided to support benefits of the introduced GDIP. Let the given $n$ compact regions to be visited by the shortest Dubins path be $\mathcal{P}=\left\{P_{1}, \ldots, P_{n}\right\}, P_{i} \in \mathbb{R}^{2}$. The solution of the DTSPN is determined as follows.

1) Determine the sequence of visits to $\mathcal{P}$ as a solution of the ETSP using centers of $P_{i} \in \mathcal{P}$, e.g., using [1].

2) For each target in the sequence, use the given disk regions and unconstrained headings as the first samples.

3) Determine the lower bound solution of the DTRP by the GDIP and use it to select promising samples.
4) Refine the selected samples and recompute the lower bound to improve the lower bound estimation.

5) Use the samples in the lower bound solution to find a feasible solution by the forward search (as in [8]).

6) Return the solution if the resolution $k_{\max }$ is reached; otherwise go to Step 4

The tight lower bound provided by the GDIP has been evaluated in randomly generated ${ }^{3}$ DTSPN instances with $n \in$ $\{10,20,50,70,100\}, \delta=1$, and $\rho=1$.

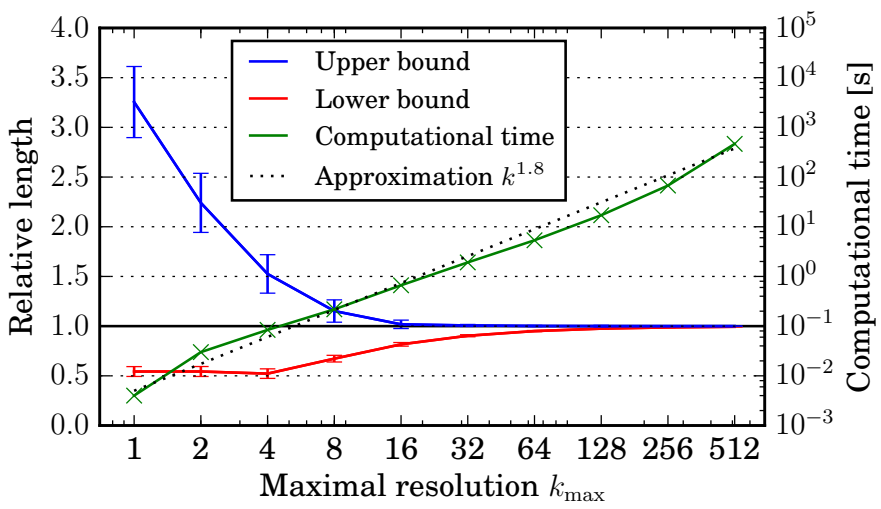

Fig. 11. Convergence of the solution cost to the lower bound and the required computational time for increasing $k_{\max }$. The solution cost is normalized by the best found solution accompanied by a computational time. The presented results are average values of 20 random problem instances for $n=10$ target regions. Note the resolution and computational time are presented in logarithmic scales.

The convergence of the DTRP solution to the lower bound has been studied for increasing the maximal number of samples $k_{\max }$. The results are depicted in Fig. 11, where the absolute solution cost is normalized to the best known solution of the particular problem instance. Based on the presented results, the computational time can be approximated by $\mathcal{O}\left(n k_{\max }^{1.8}\right)$ which is mainly because of informed sampling of the regions and heading intervals.

\section{CONCLUSION}

In this paper, we introduce the Generalized Dubins Interval Problem (GDIP) and provides its optimal solution based on the transformation of the GDIP to the OS-GDIP. Both forward and backward transformations are provided together with proofs of their correctness. Moreover, the benefits of the GDIP to the solution of the DTSPN with known sequence of the visits to the targets (called the DTRP) are demonstrated by providing a tight lower bound of the solution cost in the sampling-based solution of the DTRP. Besides, the provided lower bound allows to determine the solution quality of the DTRP (and thus the DTSPN). The reported results indicate it is possible to find a solution of the DTRP with 10 overlapping disk regions and the solution quality is around 1\% from the optimum in less than 10 seconds using a single core of a conventional processor.

\footnotetext{
${ }^{3}$ Instances have been generated with the relative density $d=0.3$ and possibly overlapping regions where the centers are randomly placed in the square bounding box with the size $s=\rho \sqrt{n} / d$.
} 


\section{REFERENCES}

[1] David Applegate, Robert Bixby, Vašek Chvátal, William Cook, Daniel Espioza, Marcos Goycoolea, and Keld Helsgaun. CONCORDE TSP Solver, 2003. URL https://www.tsp.gatech.edu/concorde.html. [cited 25 May 2018].

[2] Jean-Daniel Boissonnat, Philippe Sou Eres, and JeanPaul Laumond. The Shortest Path Synthesis for Nonholonomic Robots Moving Forwards In INRIA, NiceSophia-Antipolis, Research Report 2153. Citeseer, 1993. URL http://citeseerx.ist.psu.edu/viewdoc/summary?doi= 10.1.1.34.524

[3] Xuan-Nam Bui, Philippe Soueres, Jean-Daniel Boissonnat, and Jean-Paul Laumond. Shortest path synthesis for Dubins non-holonomic robot In International Conference on Robotics and Automation. IEEE, 1994. doi: 10.1109/ROBOT.1994.351019. URL http://ieeexplore. ieee.org/document/351019.

[4] Izack Cohen, Chen Epstein, and Tal Shima. On the discretized Dubins Traveling Salesman Problem. IISE Transactions, 49(2):238-254, 2017. doi: 10.1080/ 0740817X.2016.1217101. URL http://www.tandfonline. com/doi/abs/10.1080/0740817X.2016.1217101.

[5] Lester E Dubins. On curves of minimal length with a constraint on average curvature, and with prescribed initial and terminal positions and tangents. American Journal of Mathematics, pages 497-516, 1957. doi: 10.2307/ 2372560. URL http://www.jstor.org/stable/2372560.

[6] Jan Faigl and Petr Váňa. Self-Organizing Map for the Curvature-Constrained Traveling Salesman Problem. In International Conference on Artificial Neural Networks, pages 497-505. Springer, 2016. doi: 10.1007/ 978-3-319-44781-0_59. URL https://link.springer.com/ chapter/10.1007\%2F978-3-319-44781-0_59

[7] Jan Faigl and Petr Váňa. Unsupervised learning for surveillance planning with team of aerial vehicles. In International Joint Conference on Neural Networks (IJCNN), pages 4340-4347. IEEE, 2017. doi: 10.1109/ IJCNN.2017.7966405. URL http://ieeexplore.ieee.org/ document/7966405/

[8] Jan Faigl, Petr Váňa, Martin Saska, Tomáš Báča, and Vojtěch Spurný. On solution of the Dubins touring problem. In European Conference on Mobile Robots (ECMR), pages 1-6. IEEE, 2017. doi: 10.1109/ECMR. 2017.8098685. URL http://ieeexplore.ieee.org/document/ 8098685

[9] Xavier Goaoc, Hyo-Sil Kim, and Sylvain Lazard. Bounded-curvature shortest paths through a sequence of points using convex optimization. SIAM Journal on Computing, 42(2):662-684, 2013. doi: 10.1137/100816079. URL http://epubs.siam.org/doi/abs/10.1137/100816079.

[10] Douglas Guimaraes Macharet, Armando Alves Neto, Vilar Fiuza da Camara Neto, and Mario Montenegro Campos. An evolutionary approach for the Dubins' traveling salesman problem with neighborhoods In Proceed- ings of the 14th Annual Conference on Genetic and Evolutionary Computation, pages 377-384. ACM, 2012. doi: 10.1145/2330163.2330218. URL https://dl.acm.org/ citation.cfm?doid=2330163.2330218

[11] Karl Damkjær Hansen and Anders La Cour-Harbo. Waypoint planning with Dubins curves using genetic algorithms. In European Control Conference (ECC), pages 2240-2246. IEEE, 2016. doi: 10.1109/ECC. 2016.7810624. URL http://ieeexplore.ieee.org/document/ 7810624

[12] Jason T Isaacs and Joao P Hespanha. Dubins traveling salesman problem with neighborhoods: a graph-based approach. Algorithms, 6(1):84-99, 2013. doi: 10.3390/ a6010084. URL http://www.mdpi.com/1999-4893/6/1/ 84

[13] Jason T. Isaacs, Daniel J. Klein, and João Pedro Hespanha. Algorithms for the Traveling Salesman Problem with Neighborhoods Involving a Dubins Vehicle In American Control Conference, pages 1704-1709. IEEE, 2011. doi: 10.1109/ACC.2011.5991501. URL http: //ieeexplore.ieee.org/document/5991501

[14] Pantelis Isaiah and Tal Shima. Motion planning algorithms for the Dubins Travelling Salesperson Problem. Automatica, 53:247-255, 2015. doi: 10.1016/j. automatica.2014.12.041. URL https://www.sciencedirect. com/science/article/pii/S0005109814006207

[15] Jerome Le Ny and Eric Feron. An approximation algorithm for the curvatureconstrained traveling salesman problem In Proceedings of the 43rd Annual Allerton Conference on Communications, Control and Computing, pages 620-9, 2005. URL http://citeseerx.ist.psu.edu/ viewdoc/summary?doi=10.1.1.174.5862.

[16] Jerome Le Ny, Emilio Frazzoli, and Eric Feron. The curvature-constrained traveling salesman problem for high point densities. In 46th Conference on Decision and Control, pages 5985-5990. IEEE, 2007. doi: 10.1109/CDC.2007.4434503. URL http://ieeexplore.ieee. org/document/4434503.

[17] Jerome Le Ny, Eric Feron, and Emilio Frazzoli. On the Dubins Traveling Salesman Problem Transactions on Automatic Control, 57(1):265-270, 2012. doi: 10.1109/TAC.2011.2166311. URL http://ieeexplore.ieee. org/document/6004813.

[18] Jae-Ha Lee, Otfried Cheong, Woo-Cheol Kwon, Sung Yong Shin, and Kyung-Yong Chwa. Approximation of Curvature-Constrained Shortest Paths through a Sequence of Points. In European Symposium on Algorithms, pages 314-325. Springer, 2000. doi: 10. 1007/3-540-45253-2_29. URL https://link.springer.com/ chapter/10.1007\%2F3-540-45253-2_29.

[19] Xiang Ma and David A Castanon. Receding horizon planning for Dubins traveling salesman problems. In 45th Conference on Decision and Control, pages 5453-5458. IEEE, 2006. doi: 10.1109/CDC.2006.376928. URL http: //ieeexplore.ieee.org/document/4177966.

[20] Douglas G Macharet and Mario FM Campos. An 
Orientation Assignment Heuristic to the Dubins Traveling Salesman Problem. In Advances in Artificial Intelligence-IBERAMIA 2014, pages 457-468. Springer, 2014. doi: 10.1007/978-3-319-12027-0_ 37. URL https://link.springer.com/chapter/10.1007\% 2F978-3-319-12027-0_37.

[21] Douglas G Macharet, Armando Alves Neto, Vilar F da Camara Neto, and Mario FM Campos. Nonholonomic path planning optimization for Dubins' vehicles. In International Conference on Robotics and Automation (ICRA), pages 4208-4213. IEEE, 2011. doi: 10.1109/ ICRA.2011.5980239. URL http://ieeexplore.ieee.org/ document/5980239.

[22] Douglas G Macharet, Armando Alves Neto, Vilar F da Camara Neto, and Mario FM Campos. Data gathering tour optimization for Dubins' vehicles. In Congress on Evolutionary Computation (CEC), pages 1-8. IEEE, 2012. doi: 10.1109/CEC.2012.6256477. URL http: //ieeexplore.ieee.org/document/6256477.

[23] Douglas G Macharet, Armando Alves Neto, Vilar F da Camara Neto, and Mario FM Campos. Efficient target visiting path planning for multiple vehicles with bounded curvature In International Conference on Intelligent Robots and Systems (IROS), pages 3830-3836. IEEE, 2013. doi: 10.1109/IROS.2013.6696904. URL http://ieeexplore.ieee.org/document/6696904/.

[24] Satyanarayana Manyam and Sivakumar Rathinam. On Tightly Bounding the Dubins Traveling Salesman's Optimum. Presented at the International Symposium on Mathematical Programming, 2015. URL http://arxiv.org/ abs/1506.08752.

[25] Satyanarayana Manyam, Sivakumar Rathinam, David Casbeer, and Eloy Garcia. Shortest Paths of Bounded Curvature for the Dubins Interval Problem arXiv preprint, 2015. URL http://arxiv.org/abs/1507.06980.

[26] Satyanarayana Manyam, Sivakumar Rathinam, and David Casbeer. Dubins paths through a sequence of points: Lower and upper bounds In International Conference on Unmanned Aircraft Systems (ICUAS), pages 284-291. IEEE, 2016. doi: 10.1109/ICUAS. 2016.7502623. URL http://ieeexplore.ieee.org/document/ 7502623 .

[27] Satyanarayana G. Manyam, Sivakumar Rathinam, David Casbeer, and Eloy Garcia. Tightly Bounding the Shortest Dubins Paths Through a Sequence of Points. Journal of Intelligent \& Robotic Systems, pages 1-17, 2017. doi: 10.1007/s10846-016-0459-4. URL http://dx.doi.org/10. 1007/s10846-016-0459-4.

[28] André César Medeiros and Sebastián Urrutia. Discrete optimization methods to determine trajectories for Dubins' vehicles. Electronic Notes in Discrete Mathematics, 36:17-24, 2010. doi: 10.1016/j.endm.2010.05. 003. URL https://www.sciencedirect.com/science/article/ pii/S1571065310000041.

[29] Karl Obermeyer, Paul Oberlin, and Swaroop Darbha. Sampling-based roadmap methods for a visual reconnais- sance UAV. In AIAA Guidance, Navigation, and Control Conference, page 7568, 2010. doi: 10.2514/6.2010-7568. URL https://arc.aiaa.org/doi/abs/10.2514/6.2010-7568.

[30] Karl J Obermeyer. Path planning for a UAV performing reconnaissance of static ground targets in terrain. In AIAA Guidance, Navigation, and Control Conference, pages 10-13, 2009. doi: 10.2514/6.2009-5888. URL https://arc.aiaa.org/doi/abs/10.2514/6.2009-5888.

[31] Karl J Obermeyer, Paul Oberlin, and Swaroop Darbha. Sampling-based path planning for a visual reconnaissance unmanned air vehicle. Journal of Guidance, Control, and Dynamics, 35(2):619-631, 2012. doi: 10. 2514/1.48949. URL http://arc.aiaa.org/doi/abs/10.2514/ 1.48949 .

[32] Robert Pěnička, Jan Faigl, Petr Váňa, and Martin Saska. Dubins Orienteering Problem. Robotics and Automation Letters, 2(2):1210-1217, 2017. doi: 10.1109/LRA. 2017.2666261. URL http://ieeexplore.ieee.org/document/ 7847413 .

[33] Ketan Savla, Emilio Frazzoli, and Francesco Bullo. On the point-to-point and traveling salesperson problems for Dubins' vehicle. In Proceedings of the American Control Conference, pages 786-791. IEEE, 2005. doi: 10.1109/ACC.2005.1470055. URL http://ieeexplore.ieee. org/document/1470055.

[34] Petr Váňa and Jan Faigl. On the Dubins Traveling Salesman Problem with Neighborhoods. In International Conference on Intelligent Robots and Systems (IROS), pages 4029-4034. IEEE, 2015. doi: 10.1109/IROS. 2015.7353945. URL http://ieeexplore.ieee.org/document/ 7353945

[35] Xin Yu and J.Y. Hung. A genetic algorithm for the Dubins Traveling Salesman Problem. In International Symposium on Industrial Electronics, pages 1256-1261. IEEE, 2012. doi: 10.1109/ISIE.2012.6237270. URL http://ieeexplore.ieee.org/document/6237270

[36] Xin Yu and JY Hung. Optimal path planning for an autonomous robot-trailer system In Industrial Electronics Society, 38th Annual Conference on (IECON), pages 2762-2767. IEEE, 2012. doi: 10.1109/IECON. 2012.6389140. URL http://ieeexplore.ieee.org/document/ 6389140.

[37] Xing Zhang, Jie Chen, Bin Xin, and Zhihong Peng. A memetic algorithm for path planning of curvatureconstrained UAVs performing surveillance of multiple ground targets. Chinese Journal of Aeronautics, 27(3):622-633, 2014. doi: 10.1016/j.cja.2014.04. 024. URL https://www.sciencedirect.com/science/article/ pii/S1000936114000946. 